\title{
Treatment Strategies and Recommendations for Pseudomonas aeruginosa Infections
}

\author{
NICHOLAS M MOORE, MARIBETH L FLAWS
}

Learning Objectives

1. Describe the mechanism of action of the primary anti-pseudomonal agents.

2. List the advantages and disadvantages of combination drug therapy.

3. List the advantages and disadvantages of mono drug therapy.

4. Determine which antibiotic combinations are appropriate for treatment of various infections caused by Pseudomonas aeruginosa.

ABBREVIATIONS: DNA = Deoxyribonucleic Acid, IDSA = Infectious Disease Society of America, ATS = American Thoracic Society.

INDEX TERMS: Pseudomonas aeruginosa, antiPseudomonas antibiotics, Beta Lactams.

Clin Lab Sci 2011;24(1):52

Nicholas M. Moore, MS, MLS(ASCP), Rush University, Department of Medical Laboratory Science, Chicago, IL

Maribeth L. Flaws, PhD, SM(ASCP)SI, Rush University, Department of Medical Laboratory Science, Chicago, $I L$

Address for Correspondence: Nicholas M. Moore, MS, MLS (ASCP), Department of Medical Laboratory Science, 600 S. Paulina St., Suite 1014, Chicago, IL 60612, 312942-2111, Fax: (312) 942-6464, Nicholas_Moore@ rush.edu

Pseudomonas aeruginosa is a common cause of infection, especially hospital-acquired infections. It is opportunistic, more often causing infection in immunocompromised hosts and it is often antibiotic resistant, complicating the efficacy of therapy. These factors have increased the number of patients infected with $P$. aeruginosa, increased the severity of those infections, and caused a great deal of conflict amongst infectious disease practitioners on how to appropriately and effectively manage their treatment. The following sections will describe appropriate treatment regimens for non-cystic fibrosis patients with infections caused by $P$. aeruginosa and discuss empiric as well as mono versus combination therapy protocols.

Anti-Pseudomonas Antibiotics

The antimicrobial agents listed in Table 1 have activity against $P$. aeruginosa.

Table 1. Antimicrobial agents with activity against Pseudomonas aeruginosa grouped by class.
Antibiotic Class

Penicillins

Penicillin/Beta-

lactamase

inhibitor

Cephalosporins

Monobactams

Carbapenems

Fluoroquinolones

Aminoglycosides

Polymyxins

\section{Drug ticarcillin, penicillin}

ticarcillin-clavulanate, piperacillin-tazobactam

ceftazidime, cefepime

aztreonam

imipenem-cilastatin, meropenem

ciprofloxacin, levofloxacin block DNA

gentamicin, tobramycin amikacin

colistin synthesis (DNA gyrase)

Mechanism of Action inhibit bacterial cell wall synthesis

inhibit bacterial cell wall synthesis

inhibit bacterial cell wall synthesis

inhibit bacterial cell wall synthesis

inhibit bacterial cell wall synthesis

inhibition of protein synthesis

disrupts outer cell membrane 


\section{FOCUS: PSEUDOMONAS AERUGINOSA}

\section{Beta Lactams}

Beta-lactams are a broad group of antibiotics that include penicillins, cephalosporins, monobactams and carbapenems. At the molecular level, the beta-lactams have thiazolidine and beta-lactam rings to which is attached a side chain. Beta-lactams are one of the most widely prescribed antibiotic agents in the United States. They are bactericidal and act by binding to transpeptidases [also known as penicillin-binding proteins (PBP)] thereby interfering with the synthesis of the peptidoglycan layer of the cell wall. Bacteria, including $P$. aeruginosa, become resistant to beta-lactams by either possessing enzymes (beta-lactamases) that hydrolyze the beta-lactam ring or altering the PBPs such that the antimicrobial agent can no longer bind and exert its effect.

The beta-lactams that have the most activity against $P$. aeruginosa include piperacillin, ticarcillin, $3^{\text {rd }}$ and $4^{\text {th }}$ generation cephalosporins (ceftazidime and cefepime respectfully), and carbapenems like imipenem or meropenem.

\section{Quinolones}

The quinolones (e.g. nalidixic acid) and fluoroquinolones (e.g. ciprofloxacin) are broad-spectrum synthetic antimicrobial agents. They are bactericidal drugs that interfere with DNA replication. Specifically, they inhibit the activity of DNA gyrase and topoisomerase IV. DNA gyrase is the enzyme that unwinds the double-stranded DNA and topoisomerase IV decatenates DNA following replication relaxing positive supercoils. There are three known mechanisms of resistance to quinolones. Efflux pumps decrease the intracellular quinolone concentration, plasmid-encoded resistance genes produce proteins that bind to DNA gyrase, protecting it from the quinolones, and finally, mutations in DNA gyrase or topoisomerase IV decrease their binding affinity to quinolones, decreasing the drug's effectiveness.

The fluoroquinolones that have anti-pseudomonal activity include ciprofloxacin, levofloxacin and ofloxacin.

\section{Aminoglycosides}

Aminoglycosides are the final group of antibiotics with activity against $P$. aeruginosa and are derived from
Streptomyces. They function by inhibiting protein synthesis either by binding to the $30 \mathrm{~S}$ or $50 \mathrm{~S}$ ribosomal subunit. Depending on the dosage, aminoglycosides can be bacteriostatic or bactericidal agents. Due to the narrow therapeutic range of the aminoglycosides, the plasma drug level must be continuously monitored in patients to ensure that the dosage remains in the therapeutic range. This class is also one of the few in which susceptibility has not been drastically reduced when used for Pseudomonas, Acinetobacter and Enterobacter species. In spite of this, physicians may still be hesitant to use them unless absolutely needed due to the relative frequency of associated ototoxicity or nephrotoxicity. Resistance to the aminoglycosides occurs as a result of the production of enzymes that degrade the aminoglycosides or by actively pumping the drug out of the cell.

The aminoglycosides that are used to treat infections caused by $P$. aeruginosa are tobramycin, amikacin, and gentamicin. These agents are typically used in combination therapy with another antimicrobial agent and are only recommended as the sole form of therapy for urinary tract infections caused by $P$. aeruginosa.

\section{Empiric Therapy}

When a bacterial infection is suspected, cultures are performed and empiric therapy is initiated well before the final identification and susceptibility results are back from the microbiology laboratory. Typically, empiric regimens are decided based on the geographic location, patient population, types of infections most frequently encountered and whether or not a community versus nosocomial-acquired infection is suspected. One study ${ }^{1}$ examined the impact of appropriate antimicrobial therapy in cases of $P$. aeruginosa bacteremia. They based their assessment on in-hospital mortality and length of stay (LOS) examining 167 cases of bacteremia attributed to $P$. aeruginosa. 123 patients (86 percent) received appropriate empirical antibiotic therapy and sixty-one patients of the total 167 (36.5 percent) died. They found that while appropriate empirical therapy was not associated with a significant decrease in mortality, they did see a reduction in the mean LOS by 7 percent in patients who received appropriate empirical therapy as compared to those who did not. ${ }^{1}$ Some studies $^{2-4}$ have shown that there is an association 


\section{FOCUS: PSEUDOMONAS AERUGINOSA}

between adequate empiric therapy and outcomes in cases of $P$. aeruginosa infections while others ${ }^{5-8}$ reported no such association.

\section{Combination versus Monotherapy}

In cases involving infection with $P$. aeruginosa, one of the more controversial issues revolves around whether combination or monotherapy is more appropriate. There have been several retrospective studies and meta analyses published in recent years in favor of both sides. According to consensus guidelines reached by the Infectious Disease Society of America (IDSA) and the American Thoracic Society (ATS) for empiric management of community- or hospital-acquired pneumonia, the following approach is recommended in patients who have risk factors for $P$. aeruginosa: 1 ) use of an anti-pseudomonal beta-lactam plus an antipseudomonal quinolone, or 2) an anti-pseudomonal beta-lactam plus an aminoglycoside or 3) an antipseudomonal quinolone plus an aminoglycoside. Selection of antibiotics should include consideration of local antimicrobic resistance patterns as well as institutional antibiograms. The consensus suggests a recommended therapy of two weeks, but state that duration of therapy should be tailored to the patient's signs and symptoms of clinical improvement. ${ }^{9}$

One multicenter, retrospective study ${ }^{10}$ conducted by Garnacho-Montero and colleagues in 2007 sought to evaluate whether one antibiotic achieved equal outcomes compared to combination therapy in patients with ventilator-associated pneumonia (VAP) caused by $P$. aeruginosa. Empiric monotherapy was used in 67 out of 183 cases that were studied. They found that more patients on monotherapy were inappropriately treated than those who were on combination therapy. In addition, the mortality rate was higher amongst patients treated with only one drug. They concluded that combination therapy be used at least initially in order to increase the odds that at least one drug is effective before the organism identification and susceptibility are known.

Most studies favor combination therapy for initial treatment and management of $P$. aeruginosa infections. Some studies mention that after initial susceptibility results are known, clinicians cut back to one drug to which the bacteria are susceptible. This is done to prevent the development of antibiotic resistance. Fluoroquinolones, though, should be discouraged for empiric therapy. Fluoroquinolones have been heavily prescribed in recent years, and concomitantly resistance has increased. It has previously been shown that empirical use of fluoroquinolones served as a predictor of mortality in patients infected with $P$. aeruginosa because with fluoroquinolone resistance increasing, empiric treatment with a fluoroquinolone resulted in a delay in the patient receiving an antimicrobial to which the $P$. aeruginos $a$ was susceptible leading to a decrease in cure and an increase in mortality. ${ }^{11}$ This same group performed a follow-up study published in 2008 that looked at the effect of reducing the empirical prescribing of fluoroquinolones in suspected infections with $P$. aeruginosa on the rate of resistance to fluoroquinolones. ${ }^{12}$ They found that reducing the use of fluoroquinolones resulted in shortened LOS, a shortened delay for patients to receive effective therapy and a 2 -fold reduction in infection-related mortality from $22 \%$ to $9 \%$ in patients with fluoroquinoloneresistant strains of $P$. aeruginosa. Their results were consistent with other studies that showed that inadequate empirical therapy was a predictor of mortality in the critically ill. ${ }^{1,12,13}$

A study was conducted at Rush University Medical Center in 2009 that evaluated the antibiogram of $P$. aeruginosa isolated from blood cultures collected from hospitalized patients who did not have cystic fibrosis (unpublished data). The researchers were concerned about increasing resistance rates and wanted to determine how combination therapy would affect patient care. This study demonstrated that the use of an anti-pseudomonal beta-lactam with a fluoroquinolone or an aminoglycoside offered a higher probability expressed as an odds ratio that $P$. aeruginosa would be susceptible to at least one of the agents in the regimen. This was statistically significant for all combinations of beta-lactams tested with levofloxacin or one of three aminoglycosides (gentamicin, tobramycin or amikacin) with the exception of piperacillin/tazobactam with levofloxacin (Table 2). Though this last combination did not prove to be statistically significant, this combination did successfully cover 96 percent of isolates tested (Figure 1). This data would seem to sup-. 
Table 2. Mantel-Haenszel common odds ratios (OR).

\begin{tabular}{|c|c|c|c|c|c|c|c|c|}
\hline \multirow[b]{2}{*}{ Beta-lactam } & \multicolumn{2}{|c|}{ Levofloxacin } & \multicolumn{2}{|c|}{ Gentamicin } & \multicolumn{2}{|c|}{ Tobramycin } & \multicolumn{2}{|c|}{ Amikacin } \\
\hline & p-value & OR $(95 \% \mathrm{CI})$ & p-value & OR $(95 \% \mathrm{CI})$ & p-value & OR $(95 \% \mathrm{CI})$ & p-value & OR $(95 \% \mathrm{CI})$ \\
\hline $\begin{array}{l}\text { Piperacillin/ } \\
\text { Tazobactam }\end{array}$ & 0.136 & $1.574(0.867-2.858)$ & $<0.001$ & $2.994(1.618-5.538)$ & 0.004 & $2.750(1.393-5.427)$ & 0.039 & $2.220(1.040-4.739)$ \\
\hline Imipenem & $<0.001$ & $8.306(5.505-12.534)$ & $<0.001$ & $4.725(3.2-6.976)$ & $<0.001$ & $9.877(5.322-18.33)$ & $<0.001$ & $4.906(2.695-8.931)$ \\
\hline Cefepime & $<0.001$ & 7.035(4.664-10.612) & $<0.001$ & $8.057(5.311-12.223)$ & $<0.001$ & $10.779(5.874-19.779)$ & $<0.001$ & $7.055(3.785-13.148)$ \\
\hline Aztreonam & $<0.001$ & $3.442(2.273-5.211)$ & 0.002 & $1.891(1.261-2.836)$ & $<0.001$ & $3.874(2.041-7.355)$ & 0.037 & $1.914(1.042-3.516)$ \\
\hline Meropenem & $<0.001$ & $10.185(6.33-16.389)$ & $<0.001$ & $5.163(3.335-7.994)$ & $<0.001$ & $5.808(3.242-10.405)$ & $<0.001$ & $5.223(2.832-9.635)$ \\
\hline $\begin{array}{l}\text { CI: Confiden } \\
\text { Significance: }\end{array}$ & rval & & & & & & & \\
\hline
\end{tabular}

port the correlation that empiric combination therapy is associated with improved outcomes, but that was not specifically addressed in this study.

There are many reasons why combination therapy is viewed as more beneficial to the patient. These include the potential for synergy, having an increased likelihood that at least one agent will be effective, and the potential to reduce the emergence of resistant strains. There are, however, benefits associated with monotherapy that should not be dismissed. Those include lower health care costs, fewer adverse events — especially when aminoglycosides are not administered - the utilization

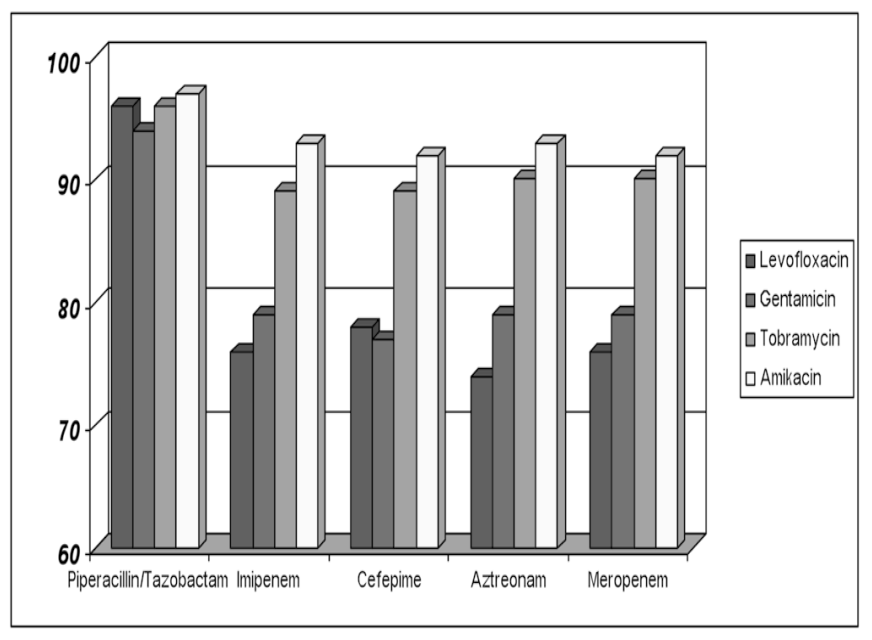

Figure 1. Combined susceptibilities (\%). This figure illustrates the percentage of isolates that were susceptible to that combination of antimicrobial agents. of a narrower spectrum of treatment and the possibility of reducing the probability the patient could develop an infection with a multidrug resistant strain of $P$. aeruginosa.

$P$. aeruginosa has been recognized as a cause of infections for almost 130 years. It is likely that $P$. aeruginosa will be causing infections in humans for another 130 years. As reviewed in this Focus series, $P$. aeruginosa will be isolated in clinical laboratories long into the future for the following reasons:

- The organism is ubiquitous in nature increasing the likelihood of exposure

- Humans are living longer with chronic disease and immunosuppression providing susceptible hosts for the organism to infect

- $P$. aeruginosa has many mechanisms for resisting being killed by antimicrobial agents and

- Antimicrobial agents are not always used judiciously and when overprescribed drive the development of resistance.

To reduce the incidence of $P$. aeruginosa, we will need to identify patients at risk for infection and prevent exposure to the organism as well as try to prevent the further development of resistance by analyzing resistance trends and adjusting therapeutic regimens regularly. Studies have shown that antimicrobial pressure leads to the development of resistance and that 


\section{FOCUS: PSEUDOMONAS AERUGINOSA}

in the absence of that pressure, the organism reverts to being susceptible. We need to continually assess resistance, recognize trends and implement change as necessary. Only then can we come close to winning the war against such a powerful adversary.

\section{REFERENCES}

1. Osih RB, McGregor JC, Rich SE, et al. Impact of empiric antibiotic therapy on outcomes in patients with Pseudomonas aeruginosa bacteremia. Antimicrob Agents Chemother 2007;51:839-44.

2. Bodey GP, Jadeja L, Elting L. Pseudomonas bacteremia: retrospective analysis of 410 episodes. Arch Intern Med 1985;145:1621-9.

3. Chamot EE, El Amari BE, Rohner P, Van Delden C. Effectiveness of combination antimicrobial therapy for Pseudomonas aeruginosa bacteremia. Antimicrob Agents Chemother 2003;47:2756-64.

4. Micek ST, Lloyd AE, Ritchie DJ, et al. Pseudomonas aeruginosa bloodstream infection: importance of appropriate initial antimicrobial treatment. Antimicrob Agents Chemother 2005; 49:1306-11.

5. Bryan CS, Reynolds KL, Brenner ER. Analysis of 1,186 episodes of gram-negative bacteremia in non-university hospitals: the effects of antimicrobial therapy. Rev Infect Dis 1983;5:629-38.

6. Ibrahim EH, Sherman G, Ward S, et al. The influence of inadequate antimicrobial treatment of bloodstream infections on patient outcomes in the ICU setting. Chest 2000;118:14655.
7. Lee SC, Hua CC, Yu TJ, et al. Risk factors of mortality for nosocomial pneumonia: importance of initial antimicrobial therapy. Int J Clin Pract 2005;59:39-45.

8. MacArthur RD, Miller M, Albertson T, et al. Adequacy of early empiric antibiotic treatment and survival in severe sepsis: experience from the MONARCS trial. Clin Infect Dis 2004;38:284-8.

9. Mandell LA, Wunderink RG, Anzueto A, et al. Infectious Diseases Society of America/American Thoracic Society consensus guidelines on the management of communityacquired pneumonia in adults. Clin Infect Dis 2007;44S:S27.

10. Garnacho-Montero J, Sa-Borges M, et al. Optimal management therapy for Pseudomonas aeruginosa ventilator-associated pneumonia: an observational, multicenter study comparing monotherapy with combination antibiotic therapy. Crit Care Med 2007;35:1888-95.

11. Hsu DI, Okamoto MP, Murthy R, Wong-Beringer A. Fluoroquinolone-resistant Pseudomonas aeruginosa: Risk factors for acquisition and impact on outcomes. J Antimicrob Chemother 2005;55:535-41.

12. Nguyen LH, Hsu DI, Ganapathy V, Shriner K, WongBeringer A. Reducing empirical use of fluoroquinolones for Pseudomonas aeruginosa infections improves outcomes. J Antimicrob Chemother. 2008;61:714-20.

13. Orbitsch MD, Fish DN, MacLaren R, Jung R. National surveillance of antimicrobial resistance in Pseudomonas aeruginosa isolates obtained from intensive care unit patients from 1993 to 2002. Antimicrob Agents Chemother. 2004;48: 4606-10. 